\title{
Clinicopathological Profile of Hodgkin Lymphoma in Tertiary Care Hospital
}

\author{
DR. P. Geetha M.D. ${ }^{1}$, Prof DR. Geetha Devadas M.D. ${ }^{2}$ \\ ${ }^{1}$ Assistant Professor, Department Of Pathology, Government Mohan Kumaramangalam Medical College, \\ Salem, India. \\ ${ }^{2}$ Professor, Department Of Pathology, Madras Medical College, India
}

\begin{abstract}
Hodgkin lymphoma is one of the most enigmatic diseases known. Our aim is to study the epidemiology of Hodgkin lymphoma including age and sex incidence and to highlight the differences in the clinical and pathological profile of Indian Hodgkin lymphoma from its western counterparts.

Materials and methods: A total of 142 cases were studied. The case records were reviewed for clinical presentation. The H\&E slides were studied to assess the morphology, presence of Reed Sternberg cells and its variants and background infiltrate.

Results: There was a bimodal age distribution noted. The age range was 9-77 years. The Male: female ratio was 1.77:1. Classical Hodgkin Lymphoma is the most common type (93\%) with mixed cellularity be the predominant subtype. Stage II disease was predominantly seen.

Conclusion: Our study highlights the differences in the clinical and pathological profile of Indian Hodgkin lymphoma from its western counterparts. Mixed cellularity was the most common subtype in contrast to western population in which nodular sclerosis is common. Distinct pattern of expression of Hodgkin lymphoma was noticed which is comparable to some of the Indian studies and different from other western studies.
\end{abstract}

Keywords: epidemiology, Hodgkin lymphoma, staging,

\section{Introduction}

Hodgkin lymphoma is a lymphoproliferative disorder, accounting for about $1 \%$ of all tumours occurring throughout the year worldwide[1]. It constitutes about $30 \%$ of all lymphomas. There is a wide international variation in the incidence of Hodgkin lymphoma. The greatest incidence rates are seen in Southern Europe and Northern America. In countries like Asia and Africa, incidence rates for Hodgkin lymphoma are 1 per 100,000 population, with the world average being around 1.2 per 100,000 for males and 0.8 per 100,000 for females.

Epstein Barr Virus infection has been considered as one of the most important etiological factor for Hodgkin lymphoma and the association depends on the age, subtype and the immune status of the individual. Hodgkin lymphoma can be diagnosed by the presence of typical neoplastic giant cells called Reed Sternberg cell in a background containing prominent non neoplastic inflammatory infiltrate.

Hodgkin lymphoma can be broadly classified into Classical (CHL) and Nodular lymphocyte predominant type (NLPHL). The CHL is the most common type which includes Nodular sclerosis (NS), Mixed cellularity (MC), Lymphocyte rich (LR) and Lymphocyte depleted (LD).

\section{Aims And Objectives}

To study the epidemiology including age and sex incidence and distribution of Hodgkin lymphoma in a tertiary care hospital and to study the clinicopathological profile, histo-morphological features and various subtypes of Hodgkin lymphoma.

\section{Material And Methods}

A total number of 142 cases were studied from tertiary care hospital, Chennai. Detailed history of the cases regarding age, sex, history, type of procedure, site, size, stage, HIV status, previous biopsy and FNAC including treatment history in a case of relapse were obtained for all the 142 lymph node cases reported during the period of study from surgical pathology records. Hematoxylin and Eosin stained $4 \mu$ thick sections of the paraffin tissue blocks of specimens were reviewed. The following clinical and pathological parameters were evaluated, Age, gender,clinical features, hepatomegaly, splenomegaly and histological subtype. The staging was done by Ann Arbor staging system.

\section{Observation And Results}

Out of 1273 cases of lymph node malignancies, 142 were Hodgkin lymphoma cases. The youngest age reported in this study was 9 years and the oldest age of presentation was 77 years. There was a bimodal age 
distribution seen, the first peak occurring in the age group of 11-20 years and the second peak in the older age group more than 50 years. Overall males were predominantly involved with the percentage of $63 \%$ and females constitute $37 \%$. B symptoms were present in 38 cases which constitutes $27 \%$. Hepatomegaly was seen in 14 cases. Splenomagaly was seen in 17 cases $(11.97 \%)$ of patients. Bone marrow infiltration was seen in 12 cases which accounts for $8.45 \%$.

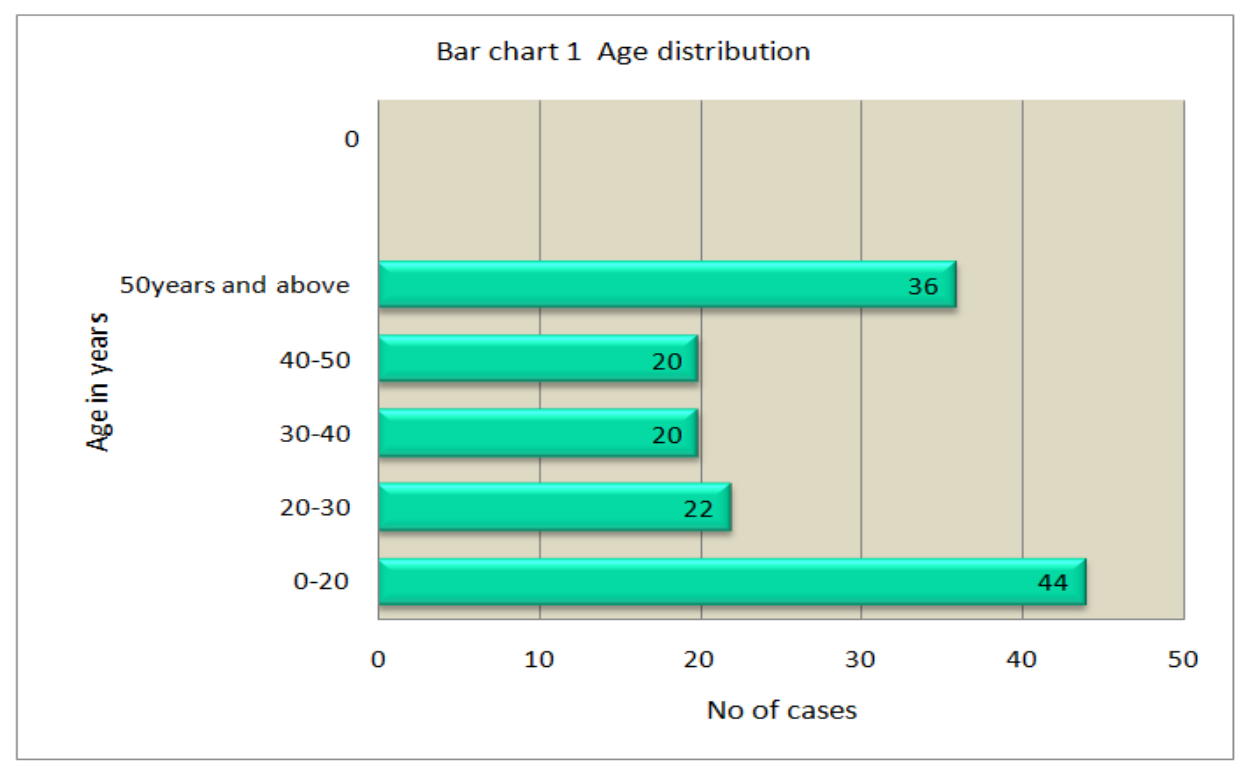

Mixed cellularity was the most common subtype which accounts for $69 \%$ followed by Nodular sclerosis. Lymphocyte depleted was the least common subtype. The majority of cases of mixed cellularity subtype occurred in the age group of 15 - 35 years followed by 35- 55 years. Mixed cellularity was most common in males and Nodular sclerosis was predominantly seen in females.

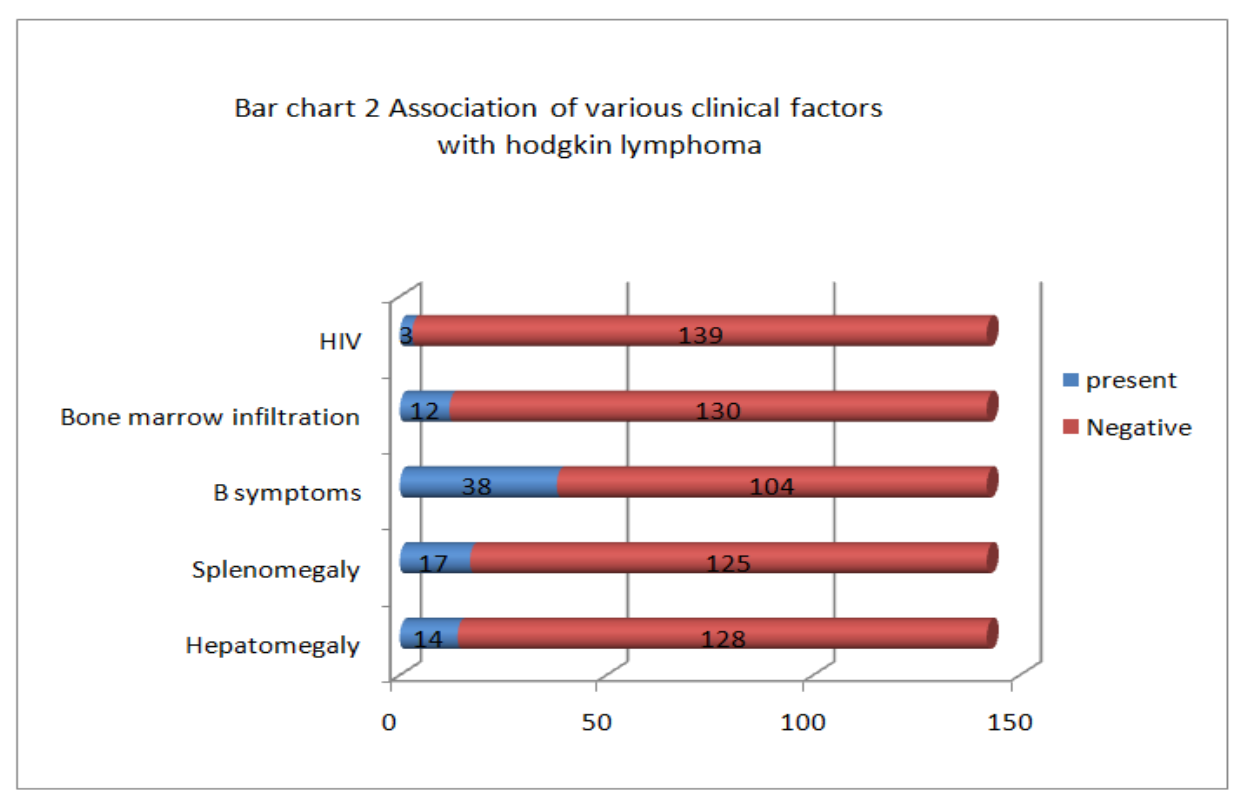

Table 1 Distribution of histological subtype in hodgkin lymphoma

\begin{tabular}{|l|l|l|}
\hline SUBTYPE & NUMBER OF CASES & PERCENTAGE \\
\hline Mixed cellularity & 98 & $69.01 \%$ \\
\hline Nodular sclerosis & 22 & $15.49 \%$ \\
\hline Lymphocyte rich & 6 & $4.23 \%$ \\
\hline Lymphocyte depleted & 2 & $1.41 \%$ \\
\hline Nodular lymphocyte predominant & 10 & $7.04 \%$ \\
\hline Unclassified & 4 & $2.82 \%$ \\
\hline Total & 142 & $100 \%$ \\
\hline
\end{tabular}


The most common presentation was Stage II disease followed by Stage III disease. B symptoms were predominantly seen in stage III and Stage IV. In this study, most of the cases with bone marrow infiltration presented with B symptoms.

Table 2 Distribution of disease stage in hodgkin lymphoma

\begin{tabular}{|l|l|l|}
\hline Stage & Number of cases & Percentage \\
\hline I & 23 & $16.20 \%$ \\
\hline II & 80 & $56.34 \%$ \\
\hline III & 27 & $19.01 \%$ \\
\hline IV & 12 & $8.45 \%$ \\
\hline TOTAL & 142 & $100 \%$ \\
\hline
\end{tabular}

\section{Discussion}

Hodgkin lymphoma showed a peak incidence in the age group of 11 to 20 years. The oldest age of presentation was 77 years and the youngest age of presentation was 9 years. Median age at presentation is 32 years. This is in concurrence with the study conducted by Ramani et al[2], who observed the median age of 34 years and the study conducted by Abu Hassan et al[3], who observed the median age to be 30 years in the patients of Hodgkin lymphoma.

In this study there was a bimodal age distribution, with first peak in the 11-30 years and the second peak in older adults more than 55 years. This is in concurrence with the study by Sudipta Chakrabarti et al[4], Jarrett et al[5] and Bidyut Krishna et al[6]. This study is discordance with the study conducted by Neelam Siddiqui et al[7], which showed unimodal distribution. In this study males were most commonly affected than females with the percentage of $64 \%$ and $36 \%$, which is concurrence with the study by L.K.Tumwine et al[8], which showed $66 \%$ and $34 \%$. The study by Tanya Siddiqui et al[9], Chandi et al ${ }^{[10]}$ and Neelam Siddiqui et al, also showed male predominance.

In this study, mixed cellularity was the most common subtype which accounts for $69 \%$ followed by nodular sclerosis accounting for $15 \%$. Lymphocyte depleted was the least common subtype with $1.4 \%$. This is similar to the study by Neelam Siddique et al. In this study, most commonly patients presented with Stage 2 disease followed by Stage 3 disease. This is similar to the study by Neelam et al.

In the present study, B symptoms were seen in $26.76 \%$ which is comparatively less than the study conducted by Al Diab et al[11] which showed $49.3 \%$ and also by various other studies.

Table 3 Comparision of histological subtype of hodgkin lymphoma

\begin{tabular}{|l|l|l|l|l|}
\hline Studies & NLPHL & Nodular sclerosis & Mixed cellularity & Lymphocyte depleted \\
\hline Medeiros LJ et al & 6.7 & 51 & 23.8 & 3.7 \\
\hline Georgii et al & 2.7 & 57.9 & 13.9 & 0.7 \\
\hline Neelam et al & 7.3 & 19.9 & 63.8 & 1.2 \\
\hline Ramadas et al & 13.5 & 22 & 50.3 & 6.3 \\
\hline Abu e Hassan et al & 25.7 & 9.5 & 14.9 & 22 \\
\hline Al Diab et al & 14.8 & 59.2 & 22.5 & 2.8 \\
\hline Sudipta Chakrabarti et al & 14.6 & 33.3 & 45.8 & 4.2 \\
\hline Waseem Memon et al & 8 & 38 & 50 & 5 \\
\hline Present study & 7.04 & 15.49 & 69.01 & 1.41 \\
\hline
\end{tabular}

Bone marrow infiltration was seen in $8.45 \%$ in this study which is similar to the study by Sudipta Chakrabarti et al which showed $8.33 \%$ and Chandi et al which showed $9.4 \%$. This is very much less than the study by Ayoz lone et al[12] and Hamid et al[13] which showed 30\% and 38\% respectively.

In the current study, nodular sclerosis was more common in females accounting for 59\% which is concurrence with the study conducted by Al Diab et al which showed 67.9\%. In the present study, mixed cellularity and nodular sclerosis predominantly presented with stage II disease which is similar to the study by Al Diab et al.

\section{Conclusion}

Bimodal age distribution was noted. Males are more commonly affected. Mixed cellularity is the most common subtype. Staging and typing of Hodgkin lymphomas has therapeutic and prognostic significance. A general awareness regarding the clinical presentation of lymphomas, along with usage of advanced investigations may lead to early precise diagnosis and proper management of this potentially curable disease. 


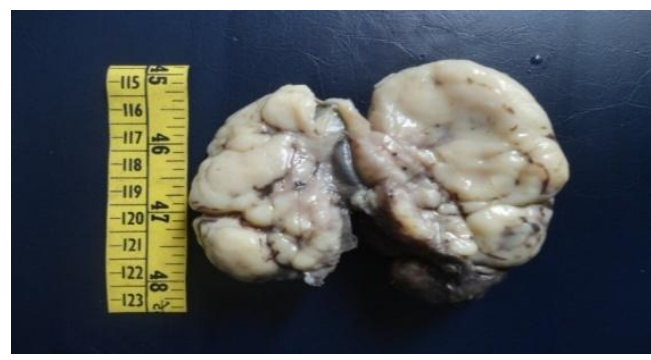

Fig 1 Nodular sclerosis - homogenous grey white with nodular cut surface.

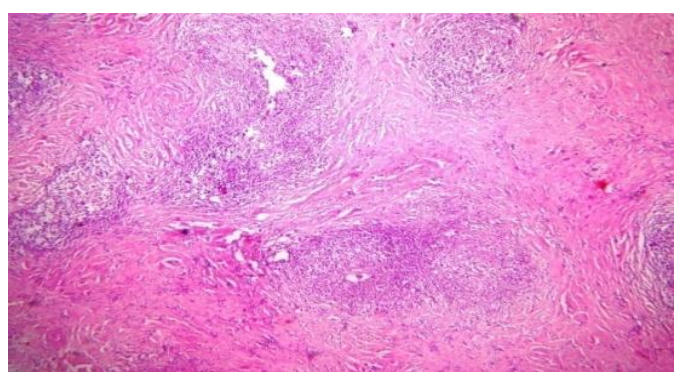

Fig 3 Nodular sclerosis showing nodules separated by fibrous bands. (H\&E 10X)

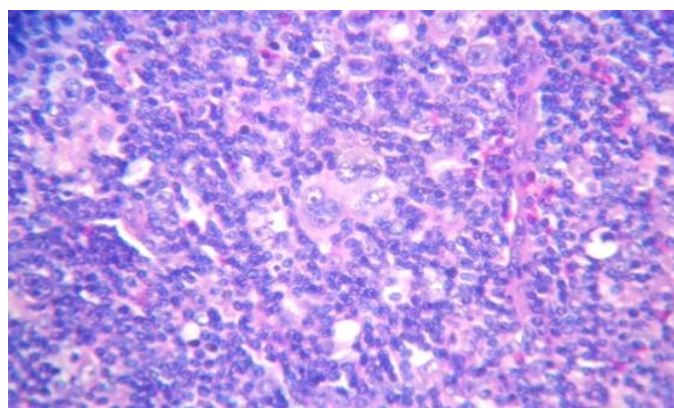

Fig 5 Nodular Lymphocyte predominant type showing popcorn cells (H\&E 10X)

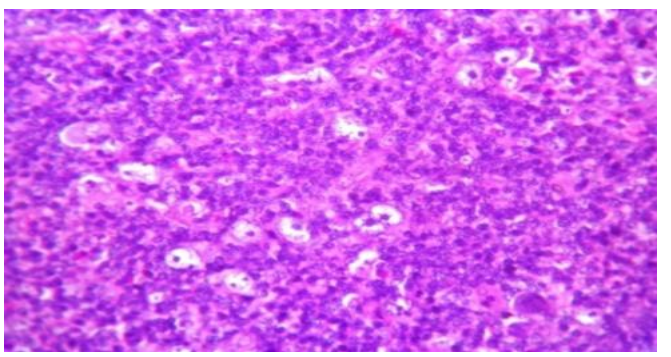

Fig 2 Mixed cellularity type showing binucleate \& mononuclear R-S cells. (H\&E 10X)

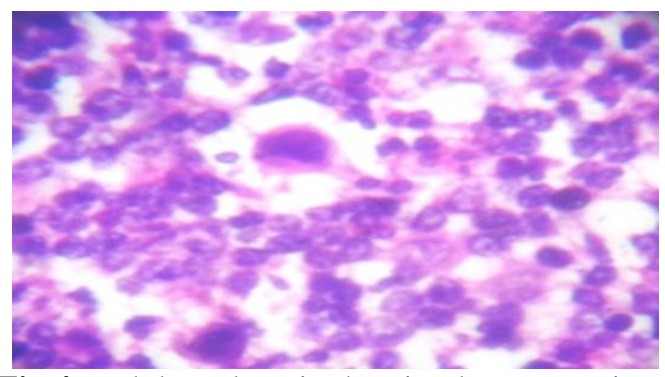

Fig 4 Nodular sclerosis showing lacunar variant of R-S cells. (H\&E 40X)

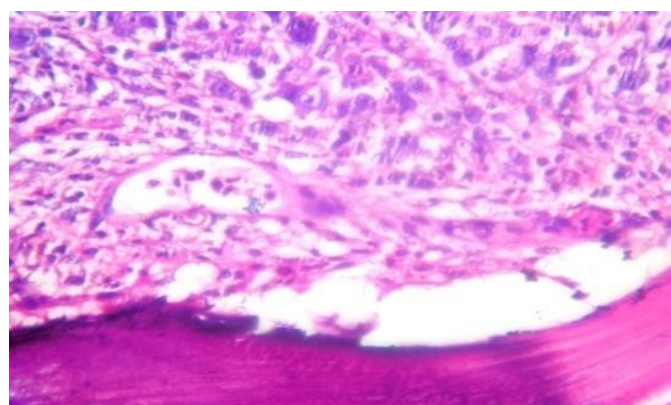

Fig 6 Shows bone marrow infiltration by Hodgkin lymphoma (H\&E 10X)

\section{References}

[1]. Pier Paolo Piccaluga, Claudio Agostinelli, Anna Gazzola, Claudio Tripodo,Francesco Bacci, Elena Sabattini, Maria Teresa Sista, ClaudiaMannu,Maria Rosaria Sapienza, Maura Rossi, Maria Antonella Laginestra,Review Article.Pathobiology of Hodgkin Lymphoma .Advances in HematologyVolume 2011, Article ID 920898, 18 pages.

[2]. Ramani A, Kumar K A, Rao K K,et al. clinicopathological profile of lymphomas in south India: A prospective rural referral hospital study of 103 cases. J Assoc physicians India 1991; 39:322-5.

[3]. Abu El Hassan MS. Ahmed ME, A/Fatah- A/Gadir, et al. Differences in presentation of Hodgkin's disease in Sudan and Western countries. TropGeogr-Med, 1993; 45:28-29.

[4]. Sudipta chakrabarthi, Supriya Sarkar, Bidyut Krishna Goswami, et al. Hodgkin and Non Hodgkin lymphomas in an Indian rura medical Institution: Comparative Clinicopathological analysis. Asian Pacific J cancer prev 2010; 11:1605-1608.

[5]. R F Jarrett, A S Krajewski, B Angus,et al. The Scotland and Newcastle epidemiological study of Hodgkin's disease: impact of histopathological review and EBV status on incidence estimates. J Clin Pathol 2003;56:811-816

[6]. Bidyut Krishna Goswami,Supriya Sarkar,Sudipta Chakrabarti, et al. Clinico-pathologic profile of Hodgkin's lymphoma in a rural medical college. Indian Journal of Hematology and Blood Transfusion.2008;24(4), 166-169.

[7]. Neelam Siddiqui, Bilal Ayub, Farhana Badar, Alia Zaidi. Hodgkin's Lymphoma in Pakistan: A Clinico-epidemiological Study of 658 cases at a Cancer Center in Lahore. Asian Pacific J Cancer Prev, 7, 651-655

[8]. L. K. Tumwine.Immunohistochemical analysis of Hodgkin's disease in Kampala, Uganda. East African Medical Journal 2004;81(8).

[9]. Tanya Siddiqui,Shahid Pervez, et al. Spectrum of Hodgkin's Disease in Children and Adults: Impact of Combined Morphologic and Phenotypic approach for exclusion of "Look-alikes". JPMA 1999;49:211.

[10]. Chandi L, Kumar L, et al. Hodgkin's disease: A retrospective analysis of 15 years experience at a large referral centre. Natl Med J India 1998;11:212-7

[11]. Al-Diab AI, Siddiqui N, Sogiawalla FF, Fawzy EM (2003). The changing trends of adult Hodgkin's disease in Saudi Arabia. Saudi Med J, 24, 617-22.

[12]. Ayaz Lone and samina naeem. Frequency and pattern of bone marrow Infiltration in hodgkin's lymphoma. Biomedica 2011;27.

[13]. Hamid A.,1 Hamid A.,2 Jabbar N. Significance of Bone Marrow Biopsy in Staging of Hodgkin's Lymphoma. Special edition annals 2010;16(1). 